\title{
Switching from Allopurinol to Febuxostat: A Comparison of The Rate of Renal Functional Decline in Patients With Moderate and Severe Chronic Kidney Disease
}

\author{
Masatora Yamasaki, Motoaki Miyazono*, Maki Yoshihara, Atsuhiko Suenaga, Masato Mizuta, Makoto Fukuda, \\ Shuichi Rikitake and Yuji Ikeda \\ Department of Nephrology, Faculty of Medicine, Japan
}

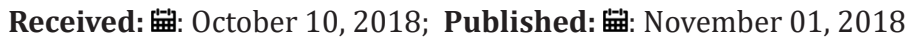

*Corresponding author: Motoaki Miyazono, Department of Nephrology, Faculty of Medicine, Japan

\begin{abstract}
Allopurinol has been widely used as a urate-lowering agent in the treatment of hyperuricemia. However, patients with renal impairment require dose reduction according to their renal function. On the other hand, strict dose adjustment is not required for the use of febuxostat and the frequency of its use is increasing. The purpose of this study was to clarify the effects of the rate of renal functional decline and other examination data by switching from allopurinol to febuxostat in patients with moderate to severe renal dysfunction. We examined the clinical records of patients treated at the Saga University Faculty of Medicine, Department of Nephrology between March 2008 and December 2016; patients who received orally-administered allopurinol for more than one year and who were able to change to febuxostat and were followed for more than two years were analyzed. The patients were divided into 3 groups: CKD(Chronic kidney desease) stage $3(n=12)$, CKD stage 4 ( $n=10)$, and CKD stage 3 and 4 combined $(n=22)$, according to the renal function at the time of switching to febuxostat. There was no significant difference in the rate of renal functional decline ( $\triangle \mathrm{eGFR}$ : $\Delta$ estimated glemerular filtration rate) in any group; however, the rate after the switch tended to be decreased in comparison to before the switch.
\end{abstract}

Keywords: Febuxostat; Allopurinol; Hyperuricemia; Chronic Kidney Disease

Abbreviations: CKD: Chronic Kidney Disease; XO: Xanthine Oxidase; Cr: Creatinine, eGFR: Estimate Glomerular Filtration Rate; BUN: Blood Urea Nitrogen; UA: Uric Acid; TP: Total Protein; Alb: Albumin; Hb: Hemoglobin; TC: Total Cholesterol; TG: Triglyceride; UP/UCr: Urinary Protein / Urine Creatinine Ratio

\section{Background}

Hyperuricemia has been reported to be related to the rapid progression of the renal functional decline in patients with chronic kidney disease (CKD) [1]. Furthermore, hyperuricemia is one of the causes of gout and kidney stone disease, and lifestyle diseases that cause cardiovascular disease, cerebrovascular disease, and arteriosclerosis. Allopurinol has been widely used as uratelowering agent in the treatment of hyperuricemia. Allopurinol reduces the uric acid concentration by acting as a xanthine oxidase (XO) inhibitor. Allopurinol is metabolized by XO and aldehyde oxidase and to oxypurinol, which is also a XO inhibitor. Oxypurinol is predominantly excreted by the kidney; thus, the risk of adverse events is higher in CKD patients. febuxostat is a novel XO inhibitor that became clinically available in Japan in 2011. Although it was reported that febuxostat was safe and effective in patients

with moderate to severe kidney dysfunction [2], few studies have examined the usefulness of switching from allopurinol to febuxostat in advanced CKD patients. The aim of the present study was to clarify the effects of the rate of renal functional decline and other factors in patients with moderate to severe renal dysfunction who switched from allopurinol to febuxostat.

\section{Patients \& Methods}

We examined the clinical records of patients who were treated in the Department of Nephrology, Saga University Faculty of Medicine from March 2008 to December 2016. Patients who received orally-administered allopurinol for more than one year and who were able to change to febuxostat and who were followed for more than two years were analyzed. The doses of allopurinol 
before switching and the dosage of febuxostat after switching were not defined. Patients with mild renal dysfunction (CKD stage 1 or 2) and those with end-stage renal failure (CKD stage 5 ) were excluded. The patients $(n=22)$ were divided into three groups according to their renal function when they switched to febuxostat: the CKD stage 3 group (S3, eGFR $30 \mathrm{ml} / \mathrm{min} / 1.73 \mathrm{~m}^{2}$ to $60 \mathrm{ml} / \mathrm{min} / 1.73 \mathrm{~m}^{2}$; $\mathrm{n}=12$ ), CKD stage 4 group (S4, eGFR $15 \mathrm{ml} / \mathrm{min} / 1.73 \mathrm{~m}^{2}$ to $30 \mathrm{ml}$ / $\min / 1.73 \mathrm{~m}^{2} ; \mathrm{n}=10$ ), CKD stage 3 and stage 4 combined group $(\mathrm{S} 3+4)$. The primary outcome was the annual rate of change in the eGFR $(\triangle \mathrm{e} G F R)$. The Wilcoxon signed rank sum test was used to compare the data before and after switching from allopurinol. $\mathrm{P}$ values of $<0.05$ were considered to indicate a significant difference. All statistical analyses were performed using the JMP Pro 12 software program.

\section{Result}

The average age of the patients in the S3 group was $53 \pm 13$

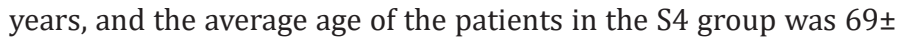

10 years. The patients in the S4 group were older than those in the S3 group. A male predominance was observed in both the S3 and S4 groups; the male to female ratio was 11:1 in the S3 group. Furthermore, the proportion of chronic glomerular nephritis as an underlying disease was high in both the S3 and S4 groups. In addition, the rate of nephrotic syndrome was high in the S3 group, while the rate of diabetic nephropathy was high in the $\mathrm{S} 4$ group. The average eGFR in the $\mathrm{S} 3$ was $41.5 \pm 7.63 \mathrm{ml} / \mathrm{min}$, while that in the S4 group was $21.3 \pm 3.84 \mathrm{ml} / \mathrm{min}$. The average eGFR in the $\mathrm{S} 3+4$ group was $32.3 \pm 11.81 \mathrm{ml} / \mathrm{min}$ (Table 1 ). The annual rates of change in the eGFR $(\triangle \mathrm{eGFR})$ of the $\mathrm{S} 3$ group in the year before switching from allopurinol to febuxostat, at one and two years after the switch are shown in Figure 1A. The $\triangle \mathrm{eGFR}$ indicates that the rate of renal functional decline was faster when the value was low. There was no significant difference after 1 year, 2 years after switching compared with before switching. We focused on the average value (Figure1B). The $\triangle \mathrm{eGFR}$ was- $1.66 \mathrm{ml} / \mathrm{min} / 1.73 \mathrm{~m}^{2} /$ year before the change to febuxostat.

Table 1: Characteristics of each groups. At switching from allopurinol to febuxostat.

\begin{tabular}{|c|c|c|c|}
\hline & Stage $\mathbf{3}(\mathbf{n}=\mathbf{1 2})$ & Stage 4 (n=10) & Stage 3+4 (n=22) \\
\hline Sex (M : F) & $11: 01$ & $06: 04$ & $63 \pm 13$ \\
\hline Age (years) & $57 \pm 13$ & $69 \pm 10$ & 9.1 \\
\hline Primary disease (\%) & & & 4.5 \\
\hline Diabetic nephropathy & 0 & 10 & 45.5 \\
\hline Nephrosclerosis & 0 & 50 & 22.7 \\
\hline Chronic glomerulonephritis & 41.6 & 0 & 18.2 \\
\hline Nephrotic syndrome & 41.6 & 20 & $1.8 \pm 0.51$ \\
\hline Others & 16.6 & $2.3 \pm 0.32$ & $32.3 \pm 11.81$ \\
\hline Cr (mg/dl) & $1.4 \pm 0.26$ & $21.3 \pm 3.84$ & $31.7 \pm 16.83$ \\
\hline eGFR (ml/min) & $41.5 \pm 7.63$ & $41.8 \pm 19.24$ & $7.7 \pm 1.09$ \\
\hline BUN (mg/dl) & $23.4 \pm 7.58$ & $7.9 \pm 1.29$ & $7.1 \pm 0.58$ \\
\hline UA (mg/dl) & $7.5 \pm 0.85$ & $7.1 \pm 0.64$ & $3.8 \pm 0.48$ \\
\hline TP (g/dl) & $7.2 \pm 0.52$ & $3.7 \pm 0.45$ & $12.6 \pm 2.34$ \\
\hline Alb (g/dl) & $3.9 \pm 0.48$ & $11.7 \pm 1.72$ & \\
\hline Hb (g/dl) & $13.2 \pm 2.53$ & $(\mathrm{n}=9) 198.2 \pm 27.84$ & $(\mathrm{n}=21) 196.1 \pm 34.3$ \\
\hline TC (mg/dl) & $194.4 \pm 38.78$ & $(\mathrm{n}=8) 155.8 \pm 85.48$ & $(\mathrm{n}=20) 166.1 \pm 77.04$ \\
\hline TG (mg/dl) & $173.9 \pm 69.02$ & $(\mathrm{n}=9) 1.1 \pm 1.2$ & $(\mathrm{n}=21) 0.8 \pm 0.97$ \\
\hline UP/Ucr (g/gCr) & $0.6 \pm 0.66$ & & \\
\hline
\end{tabular}

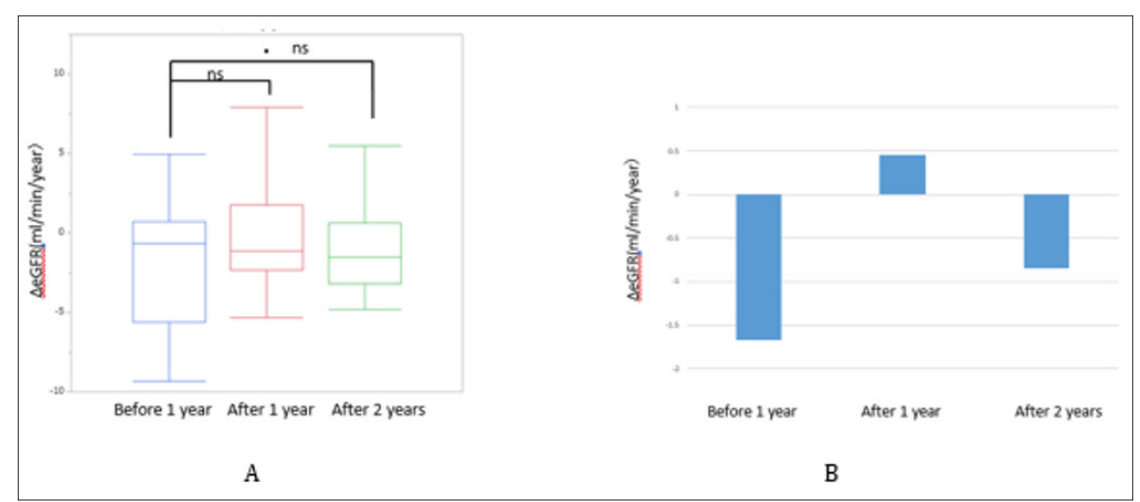

Figure 1: A: $\triangle \mathrm{eGFR}(\mathrm{ml} / \mathrm{min} /$ year) of S3 group, B: $\triangle \mathrm{eGFR}(\mathrm{ml} / \mathrm{min} /$ year) of S3 group(average). 
In contrast, the $\Delta \mathrm{eGFR}$ was $0.45 \mathrm{ml} / \mathrm{min} / 1.73 \mathrm{~m}^{2} /$ year after one year switching to febuxostat and the $\Delta \mathrm{eGFR}$ was $-0.8 \mathrm{ml} / \mathrm{min} / 1.73$ $\mathrm{m}^{2} /$ year in the two years after the switch; thus, the rate renal functional tended to decrease. The $\triangle \mathrm{eGFR}$ in the S4 group during the year before switching from allopurinol to febuxostat, and at one and two years after the switch are shown in Figure 2A. There was no significant difference after 1 year and 2 years after switching compared with before switching. Regarding average value,the $\Delta \mathrm{eGFR}$ before the switch was $-1.71 \mathrm{ml} / \mathrm{min} /$ year. The $\Delta \mathrm{eGFR}$ values at one and two years after the switch were $-1.36 \mathrm{ml} / \mathrm{min} /$ year and $-1.59 \mathrm{ml} / \mathrm{min} / 1.73 \mathrm{~m}^{2} /$ year, respectively (Figure 2B). Although there was no significant difference, the rate of renal functional decline after switching tended to be slower in comparison to before switching. The $\triangle \mathrm{eGFR}$ values of the $\mathrm{S} 3+4$ group are shown in Figure 3A. There was no significant difference after 1 year and 2 years after switching compared with before switching. Regarding average value,the $\triangle \mathrm{eGFR}$ before the switch was $-1.69 \mathrm{ml} / \mathrm{min} / 1.73 \mathrm{~m}^{2} /$ year.

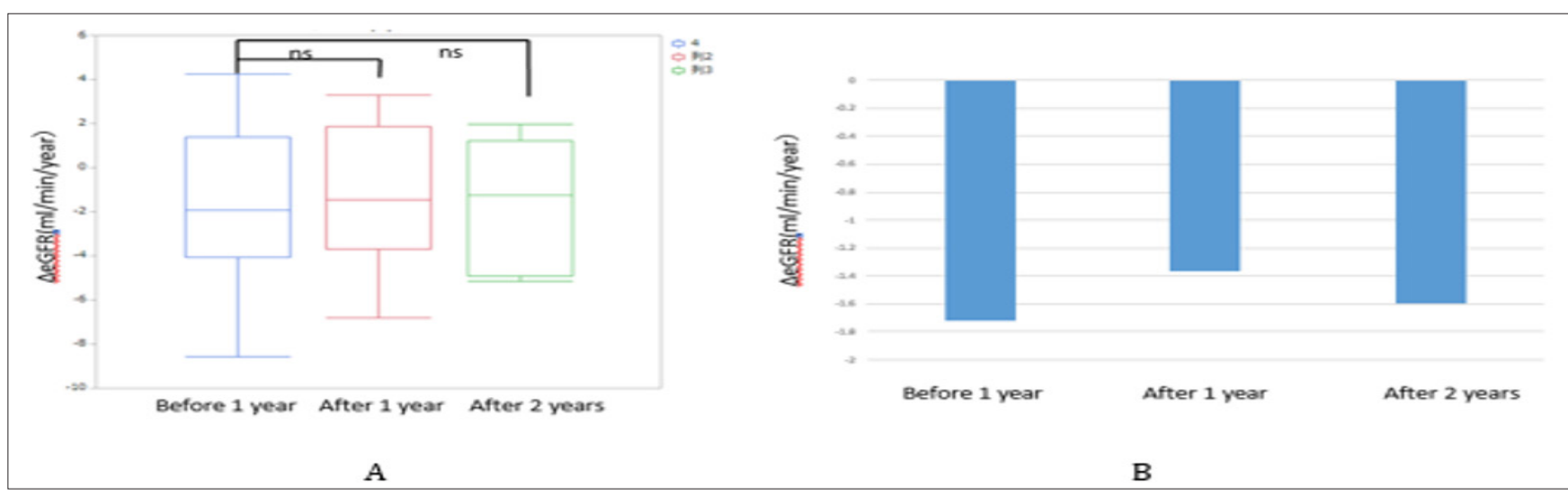

Figure 2: A: $\triangle \mathrm{eGFR}(\mathrm{ml} / \mathrm{min} /$ year) of $\mathrm{S} 4$ group, B: $\triangle \mathrm{eGFR}(\mathrm{ml} / \mathrm{min} /$ year) of $\mathrm{S} 4$ group(average).

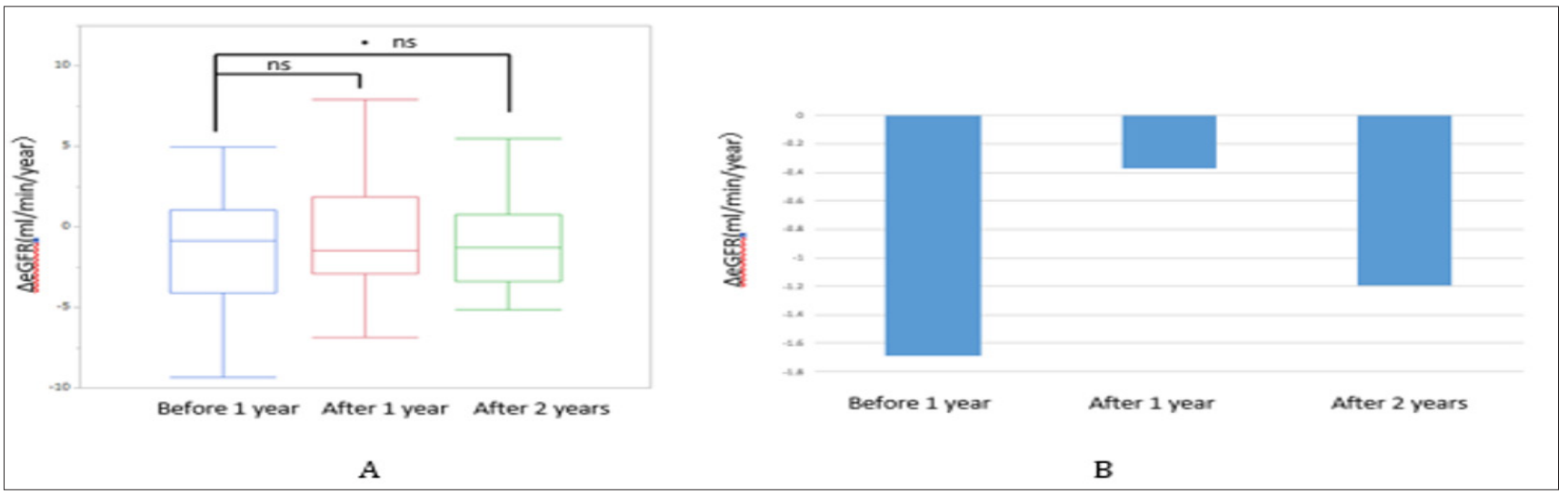

Figure 3: A: $\triangle \mathrm{eGFR}(\mathrm{ml} / \mathrm{min} /$ year) of $\mathrm{S} 3+4$ group, B: $\triangle \mathrm{eGFR}(\mathrm{ml} / \mathrm{min} /$ year) of $\mathrm{S} 4$ group(average).

The $\triangle \mathrm{eGFR}$ values at one and two years after the switch were $-0.37 \mathrm{ml} / \mathrm{min} / 1.73 \mathrm{~m}^{2} /$ year and $-1.19 \mathrm{ml} / \mathrm{min} / 1.73 \mathrm{~m}^{2} /$ year, respectively. There was no significant difference in the combined group; however, the rate of renal functional decline tended to decrease after the switch. The clinical laboratory data that showed significant changes during the observation period are shown in (Table 2). The uric acid value at two years after the switch was significantly reduced in comparison to before the switch in the S4 and $\mathrm{S} 3+4$ groups(Figure $3 \mathrm{~B}$ ). In addition, the total protein level was significantly decreased in the $\mathrm{S} 3$ and $\mathrm{S} 3+4$ groups. There were no obvious adverse events during the two years of febuxostat treatment.

\section{Discussion}

Goicoechea et al. [3] conducted a prospective, randomized trial of 113 patients with an estimated GFR (eGFR) value of $<60 \mathrm{ml} / \mathrm{min}$. Patients were randomly assigned to treatment with allopurinol $100 \mathrm{mg} /$ day $(\mathrm{n}=57)$ or to continue the usual therapy $(\mathrm{n}=56)$. The eGFR increased to $1.3 \mathrm{~mL} / \mathrm{min} / 1.73 \mathrm{~m}^{2}$ in the allopurinol group, whereas it decreased to $3.3 \mathrm{~mL} / \mathrm{min} / 1.73 \mathrm{~m}^{2}$ in the control group, and a significant difference was found between the two groups. The serum uric acid concentration reportedly decreased by $1.8 \mathrm{mg} / \mathrm{dL}$ in the allopurinol group and increased by $0.2 \mathrm{mg} /$ $\mathrm{dL}$ in the control group. Based on the results shown in Figures 1-3, although the difference was not statistically significant, the $\triangle \mathrm{eGFR}$ 
after switching to febuxostat was lower in comparison to before the switch. As shown in (Table 2), the uric acid level was significantly decreased. It was suggested that a decrease in the serum uric acid value may slow the rate of renal functional decline. Dipankar et al.

Table 2: Data with significant difference.

\begin{tabular}{|c|c|c|c|}
\hline & S3 & S4 & S3+S4 \\
\hline UA (mg/dl) & & $7.60 \rightarrow 6.95$ (after 2years) $\mathrm{p}=0.0366$ & $7.60 \rightarrow 6.75$ (after 2 years) $\mathrm{p}=0.0010$ \\
\hline TP (g/dl) & $7.45 \rightarrow 7.05$ (after 1year) $\mathrm{p}=0.0078$ & & $7.25 \rightarrow 7.00$ (after 1 year) $\mathrm{p}=0.0350$ \\
\hline
\end{tabular}

The proportion in the placebo group was 54\%, while that in the febuxostat group was $38 \%$. It was reported that febuxostat might slow the decrease in the eGFR. Furthermore, Tsuruta et al. [5], performed a 1-year cohort study of 73 hyperuricemic patients with an eGFR of $<45 \mathrm{ml} / \mathrm{min}$ who were treated with allopurinol as uratelowering therapy. In 51 patients, the treatment was changed from allopurinol to febuxostat; the other 22 patients continued to receive allopurinol. The eGFR decreased from 27.3 to $25.7 \mathrm{ml} / \mathrm{min}$ in the febuxostat group and from 26.1 to $19.9 \mathrm{ml} / \mathrm{min}$ in the allopurinol group. These reports support the possibility that switching from allopurinol to febuxostat might have reduced the rate of renal functional decline in the present study.In this study, the switching from allopurinol to febuxostat reduced the serum uric acid concentration but was not found to lead to a decrease in the urine protein excretion. However, the amount of urinary protein may be due to the result of the decreased renal function. Furthermore, the amount of total protein after the change to febuxostat was significantly decreased in the S3 and S3 + 4 groups, but not in the S4 group. Since the incidence of nephrotic syndrome in the S3 group was higher than that in the S4 group, the progression of nephrotic syndrome might have influenced the amount of total protein.

Schumacher HR Jr et al. [6] reported that the rates of adverse event rates 1072 hyperuricemic patients who received febuxostat, allopurinol, or a placebo for 28 weeks were similar. In this study, no patients experienced adverse events after switching to febuxostat [6]. If febuxostat is superior to allopurinol with regard to slowing the rate of renal functional decline, then it would seem appropriate for patients to switch from allopurinol to febuxostat.

\section{Conclusion}

In patients with moderate and severe CKD, switching from allopurinol to febuxostat may reduce the rate of renal functional

ISSN: 2574-1241

\section{DOI: 10.26717/BJSTR.2018.10.001992}

Motoaki Miyazono. Biomed J Sci \& Tech Res

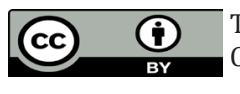

This work is licensed under Creative

Commons Attribution 4.0 License

Submission Link: https://biomedres.us/submit-manuscript.php
[4]. compared patients who were treated with febuxostat to those who received a placebo over a 6-month period in 93 patients with stage 3 or 4 CKD. The primary outcome was the proportion of patients that showed a $>10 \%$ decline in their eGFR. decline. A large-scale study with long-term observation should be performed in the future.

\section{Conflict of Interest}

The authors have no conflicts of interest directly relevant to the content of this article.

\section{References}

1. Tsai CW, Lin SY, Kuo CC, Huang CC (2017) Serum uric acid and progression of kidney disease: a longitudinal analysis and mini-review. 12: e0170393.

2. Shibagaki Y, Ohno I, Hosoya T, Kimura K (2014) Safety, efficacy and renal effect of febuxostat in patients with moderate-to-severe kidney dysfunction. Hypertens Res 37(10): 919-925.

3. Goicoechea M, de Vinuesa SG, Verdalles U, Ruiz-Caro C, Ampuero J, et al. (2010) Effect of Allopurinol in Chronic Kidney Disease Progression and Crdiovascular Risk. Clin J Am Soc Nephrol 5(8): 1388-1393.

4. Dipankar Sircar, Soumya Chatterjee, MDRajesh Waikhom, MD, DMVishal Golay, MD, DMArpita Raychaudhury, et al. (2015) Efficacy of Febuxostat for Slowing the GFR Decline in Patients With CKD and Asymptomatic Hyperuricemia: A 6-Month, Double-Blind, Randomized, PlaceboControlled Trial. Am J Kidney Dis 66(6): 945-950.

5. Yuki Tsuruta, Mochizuki $\mathrm{T}$, Moriyama $\mathrm{T}$, Itabashi $\mathrm{M}$, Takei $\mathrm{T}$, et al. (2014) Switching from allopurinol to febuxostat for the treatment of hyperuricemia and renal function in patients with chronic kidney disease. Clinical Rheumatology 33(11): 1643-1648.

6. Schumacher HR Jr, Becker MA, Wortmann RL, Macdonald PA, Hunt B, et al. (2008) Effects of febuxostat versus allopurinol and placebo in reducing serum urate in subjects with hyperuricemia and gout: a 28week, phase III, randomized, double-blind, parallel-group trial. Arthritis Rheum 59(11): 1540-1548.

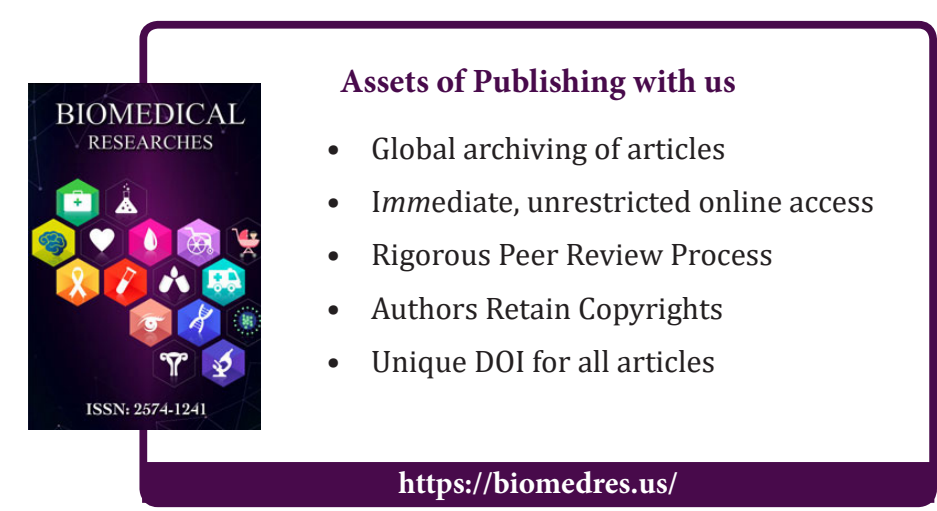

Cite this article: Masatora Yamasaki, Motoaki Miyazono, Maki Yoshihara, Atsuhiko Suenaga, Masato Mizutaet et al. Switching from Allopurinol to Febuxostat: A Comparison of The Rate of Renal Functional Decline in Patients With Moderate and Severe Chronic Kidney Disease. Biomed J Sci\&Tech Res 10(4)-2018. BJSTR. MS.ID.001992. DOI: 10.26717/ BJSTR.2018.10.001992. 